NIST Advanced Manufacturing Series 300-11

\title{
Recommendations for Collecting, Curating, and Re-Using Manufacturing \\ Data
}

\author{
Moneer Helu \\ Thomas Hedberg, Jr.
}




\title{
NIST Advanced Manufacturing Series 300-11
}

\section{Recommendations for Collecting, Curating, and Re-Using Manufacturing Data}

\author{
Moneer Helu \\ Thomas Hedberg, Jr. \\ Systems Integration Division \\ Engineering Laboratory
}

This publication is available free of charge from:

https://doi.org/10.6028/NIST.AMS.300-11

July 2020

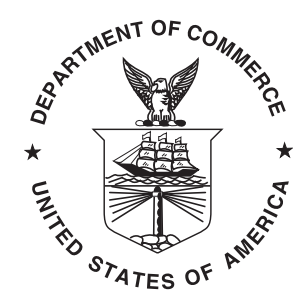

U.S. Department of Commerce

Wilbur L. Ross, Jr., Secretary

National Institute of Standards and Technology Walter Copan, NIST Director and Undersecretary of Commerce for Standards and Technology 
Certain commercial entities, equipment, or materials may be identified in this document in order to describe an experimental procedure or concept adequately. Such identification is not intended to imply recommendation or endorsement by the National Institute of Standards and Technology, nor is it intended to imply that the entities, materials, or equipment are necessarily the best available for the purpose.

National Institute of Standards and Technology Advanced Manufacturing Series 300-11

Natl. Inst. Stand. Technol. Adv. Man. Ser. 300-11, 20 pages (July 2020)

This publication is available free of charge from: https://doi.org/10.6028/NIST.AMS.300-11 


\begin{abstract}
This report provides general recommendations for collecting, curating, and re-using manufacturing data from both additive and subtractive processes. The intended audience includes researchers, technical-assessment personnel, and end-users who would like to collect data from manufacturing equipment on the shop floor. These recommendations provide guidance to design and implement a network-based solution to manage data collected from manufacturing equipment on the shop floor. To the extent possible, broadly-accepted, international, open, consensus-based standards are recommended. Using this report, the reader should be able to understand the value of collecting manufacturing data, how to get started collect it, and the relevant manufacturing data standards that may help in this process.
\end{abstract}

\title{
Key words
}

Additive manufacturing; Connectivity; Data collection; Data curation; Data management; Smart Manufacturing; Subtractive manufacturing. 


\section{Table of Contents}

1 Introduction 1

1.1 Purpose 1

1.2 Scope 1

1.3 Disclaimer 1

1.4 Overview 1

2 Motivation: Why Collect Manufacturing Data? 2

3 Connectivity: Getting Started 3

4 Overview of Relevant Standards 5

4.1 ISO 10303 (STEP) Application Protocol $242 \quad 6$

4.2 ISO/ASTM 52915 (AMF) 7

4.3 ISO 6983 (G-code) 8

4.4 ANSI MTConnect 8

4.5 ANSI/DMSC Quality Information Framework 10

4.6 ISO 32000 and ISO 14739 (PDF/PRC) 10

5 Example: National Institute of Standards and Technology (NIST) Smart Manufacturing Systems (SMS) Test Bed $\quad 11$

$\begin{array}{ll}\text { References } & 12\end{array}$

\section{List of Tables}

Table 1 Viewpoints across the product lifecycle [1]

\section{List of Figures}

Fig. 1 The challenge of maintaining multiple data collection connections when using a variety of proprietary data formats to support multiple client applications 


\section{Acronyms}

3D three-dimensional.

AMF Additive Manufacturing File Format.

ANSI American National Standards Institute.

AP Application Protocol.

ASTM American Society for Testing and Materials.

BREP boundary representation.

CAD computer-aided design.

CAI computer-aided inspection.

CAM computer-aided manufacturing.

CAx computer-aided technologies.

CMS coordinate-measurement system.

CNC computer numerical control.

DMSC Dimensional Metrology Standards Consortium.

FTO Fabrication Technology Office.

GD\&T geometric dimensions and tolerances.

HTTP Hypertext Transfer Protocol.

ISO International Standards Organization.

LOTAR long-term archival and retrieval.

MBD model-based definition.

MBE model-based enterprise.

MBM model-based manufacturing.

MQTT Message Queuing Telemetry Transport. 
NC numerical control.

NIST National Institute of Standards and Technology.

OEE overall equipment effectiveness.

OEM original equipment manufacturer.

OPC-UA Open Platform Communications United Architecture.

PDF Portable Document Format.

PMI product and manufacturing information.

PRC Product Representation Compact.

QIF Quality Information Framework.

REST Representational State Transfer.

SDO standards development organization.

SMS Smart Manufacturing Systems.

STEP STandard for the Exchange of Product Model Data.

STEP AP242 STandard for the Exchange of Product Model Data Application Protocol 242.

STL Stereolithography.

TDP technical data package.

XML Extensible Markup Language.

XMP Extensible Metadata Platform.

XSD XML Schema Definitions. 


\section{Introduction}

\subsection{Purpose}

The purpose of this document is to provide general recommendations for collecting, curating, and re-using manufacturing data. The recommendations in this document address both additive and subtractive manufacturing processes. The intended audience includes researchers, technical-assessment personnel, and end-users who would like to collect data from manufacturing equipment on the shop floor.

\subsection{Scope}

The scope of this document focuses on providing guidance to designing and implementing a network solution to collect data from manufacturing equipment on the shop floor that can then be curated and re-used for a variety of purposes. Data formats conforming to broadlyaccepted, international, open, consensus-based standards are in scope, and proprietary data formats are not in scope. Data from computer numerical control (CNC)-based subtractive processes (e.g., milling, turning) and CNC metal-based additive processes (e.g., directenergy deposition, powder-based fusion) are in scope. Data from polymer-based additive processes and mass-conserving processes (e.g., casting, forging) are not in scope.

\subsection{Disclaimer}

This work is an official contribution of the National Institute of Standards and Technology (NIST) and not subject to copyright in the United States. Certain commercial systems are identified in this paper. Such identification does not imply recommendation or endorsement by NIST. Nor does it imply that the products identified are necessarily the best available for the purpose.

\subsection{Overview}

The remainder of this document focuses on addressing three concerns:

- Why collect manufacturing data?

- How to get start collecting manufacturing data?

- What are the relevant manufacturing data standards that may help collect this data?

Finally, the NIST Smart Manufacturing Systems (SMS) Test Bed is provided an example of how the recommendations described in this document may be used to create a data connectivity solution to support the collection, curation, and re-use of manufacturing data. 


\section{Motivation: Why Collect Manufacturing Data?}

Perhaps the most fundamental use of manufacturing data is to measure, assess, and improve the performance of a manufacturing system (e.g., piece of equipment, cell, line, facility, enterprise, or supply chain) [2]. There are a variety of metrics that can be used to drive this process, including:

- Gross margin: Profit as a percentage of revenue

- Basic utilization: Percentage of total time that a piece of equipment is in cycle

- Value-added time: Percentage of cycle time that a piece of equipment is in process rather than setup

- Part-program conformance: Percentage of time that a part program is not modified by operator

- Estimation conformance: Ratio of actual to estimated cycle time for each process step

- Process efficiency: Measure of setup and run time for a job relative to a standard part or process

- Overall equipment effectiveness (OEE): Product of availability (or uptime), performance (or speed at which the piece of equipment runs as a percentage of design), and quality (or first pass yield rate)

There are also a variety of tools available to manufacturers to understand the performance of their systems, such as dashboards, analysis and reporting tools, operator feedback, and scheduling and routing systems [2]. Despite the growing availability of data and tools to use this data, many manufacturers believe that they understand their performance until confronted with real data.

The metrics reported previously represent a modest set of opportunities to improve the performance of manufacturing systems [2]. As manufacturers grow accustomed to collecting data, they often build towards higher-value use cases, such as:

- Predictive maintenance

- Prognostics

- Dynamic scheduling

- Business support (e.g., spart part provisions, request for proposals)

- Workforce augmentation and training

Supporting these use cases, though, often requires refinements to the raw data collected from a piece of equipment, including a detailed understanding of different machine states and additional context to support diagnosis and causal analysis as well as multiple viewpoints across the product lifecycle. 
Table 1. Viewpoints across the product lifecycle [1]

\begin{tabular}{|c|c|c|}
\hline Lifecycle Stage & Broad Focus & General Role \\
\hline Design & Features & $\begin{array}{l}\text { Define features to meet requirements of form, } \\
\text { fit, and function of part }\end{array}$ \\
\hline Planning & Capabilities & $\begin{array}{l}\text { Organize a set of capabilities executed through } \\
\text { different processes to create features of part }\end{array}$ \\
\hline Manufacturing & Processes & $\begin{array}{l}\text { Implement processes with maximum productiv- } \\
\text { ity to create features of part }\end{array}$ \\
\hline Inspection & Characteristics & $\begin{array}{l}\text { Compare characteristics of manufactured fea- } \\
\text { ture to its definition in design }\end{array}$ \\
\hline
\end{tabular}

Data contextualization refers to the process of combining different types of data to provide a more complete perspective of some phenomenon [1]. The quality of the information extracted from data inherently depends on the appropriateness of the context developed during data curation. The appropriate context that must be provided by data in turn depends on the viewpoint of the decision being made. For example, an operator on the shop floor and a process engineer may use the same set of data differently since they may be solving different problems and approaching these problems based on their own perspectives. Table 1 from Ref. [1] provides a general overview of the different viewpoints that exist across the product lifecycle.

The key to curating data to support multiple viewpoints is to recognize that the context needed for each viewpoint may not be uniform [1]. However, decision making in manufacturing has historically tended to focus on one viewpoint in one lifecycle stage despite the fact that decisions often impact larger portions of the product lifecycle. Thus, it is critically important to understand the use case and use it to guide the data collection and curation process. Often, the belief that more data provides more value incorrectly motivates approaching process improvements from the perspective of data rather than allowing the use case to determine the requirements and specifications of any data collection initiative.

The increasing availability of new technologies and standards associated with smart manufacturing has enabled manufactures to address more complex and valuable use cases [2]. There are many solutions available in the market today, which can make it challenging to navigate the breadth of options. The goal of Sec. 3 is provide guidance on how to select and deploy solutions for data connectivity that addresses a manufacturer's use case.

\section{Connectivity: Getting Started}

When getting started with data connectivity solutions, it is important to keep in mind that connectivity - that is collecting data from a manufacturing system in any data format using any means possible - alone is insufficient to ensure that appropriate context can be curated to address a use case. One must have a good understanding of their use case and what they hope to accomplish to ensure success in this endeavour. So, there are four fundamental 
steps that must be navigated when designing and deploying a data connectivity solution in a manufacturing system:

1. Define use case $\rightarrow$ What are the requirements?

2. Identify supported devices $\rightarrow$ What data is available?

3. Evaluate network infrastructure $\rightarrow$ How can this data be accessed and managed?

4. Execute integration activities $\rightarrow$ What support will be needed to achieve goals?

There are three questions that must be addressed when defining the use case:

1. What is the right question to ask?

2. What is the best data to use?

3. What is the appropriate context to develop?

The long-term success of a data collection activity is also predicated on developing an appropriate data management plan that enables the query or curated, contextualized data collected from the manufacturing system of interest to support the identified use case [3]. There are a number of requirements that can be included in a data management plan, including:

- General descriptions of system functions, user characteristics, and operating environments

- Identification of interfaces between uses, hardware, software, and communication systems

- Definition of key features including those tied to data curation and system administration

- Descriptions of the performance, reliability, availability, security, and maintainability needed from the system

When considering connectivity solutions, it is critically important to recognize the importance of standard data formats. Many systems may use proprietary formats, which may seem easier at the outset to use until additional data sources or client applications are introduced (see Fig. 1). At that point, it becomes clear that such systems are inherently bespoke and require tremendous expertise and cost to support multiple data formats and connections. One might consider a platform to support data connectivity, but many proprietary platforms can still lock a customer into one vendor solution, which can stifle innovation and lead to additional cost. Instead, data interoperability standards provide a flexible means for manufacturers to make strategic investments in data connectivity that limit long-term costs and ensure that the right set of solutions can be integrated as the need arises. These standards are the focus of Sec. 4.

No matter the data format that may be selected, it is important to recognize that man- 


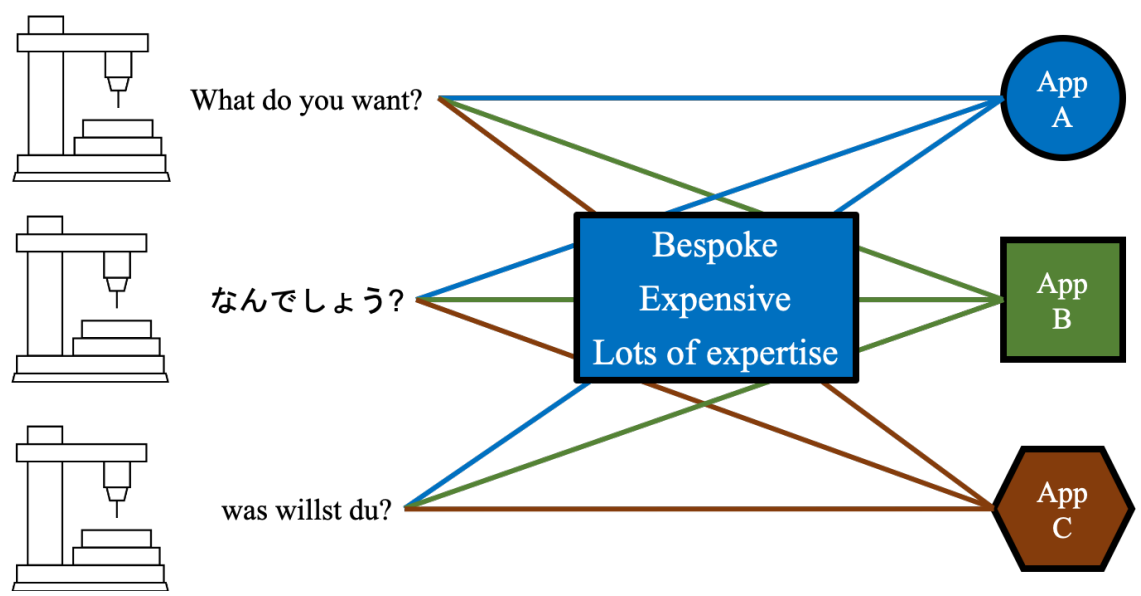

Fig. 1. The challenge of maintaining multiple data collection connections when using a variety of proprietary data formats to support multiple client applications

ufacturing systems themselves may present additional challenges when designing and deploying a data connectivity solution [3]. Specifically, these challenges include:

- Different data formats and data and communications protocols

- Need for process-related information to provide full context

- Large variety of equipment age and computational power

- Obsolete operating systems

- Large data volumes over large range of temporal scales

- Demanding limitations of physical environment

- Need for extensive time synchronization

Solutions exist to address these challenges, but it is important to keep them in mind, especially when identifying supported devices and evaluating network infrastructures so that these may be considered when defining the specifications of the data collection system. The NIST SMS Test Bed described in Sec. 5 provides some examples of these solutions.

\section{Overview of Relevant Standards}

Only internationally recognized, open, consensus-based standards were selected for review and recommendation. Standards-based data formats should be used at all times to ensure effective traceability, data-quality control, and long-term archival and retrieval (LOTAR). At a minimum, a standard must be developed by an American National Standards Institute (ANSI) recognized standards development organization (SDO) to be included in this review. A data standard is included in this review if the standard is an input to manufacturing processes (e.g., design data to a manufacturing plan) or the standard is an output from 
manufacturing processes (e.g., machine execution data, inspection data).

\subsection{ISO 10303 (STEP) Application Protocol 242}

The standard, ISO 10303-242:2014 [4] titled "Managed model-based 3D engineering," commonly known as STandard for the Exchange of Product Model Data Application Protocol 242 (STEP AP242), is an international standard that shows promise for enabling linked data. The goal of STEP AP242 is to support a manufacturing enterprise with a range of standardized information models that flow through a long and wide "digital thread" that makes the manufacturing systems in the enterprise smart [5]. Digital data plays a central role in achieving the goal of STEP AP242.

First published in December 2014, STEP AP242 contains extensions and significant updates to other STandard for the Exchange of Product Model Data (STEP) Application Protocols (APs) for product and manufacturing information (PMI), kinematics, and tessellation [6]. PMI is the presentation and representation of geometric dimensions and tolerances (GD\&T), material specifications, component lists, process specifications, and inspection requirements within a three-dimensional (3D) product definition [7]. Reference [7], comparing drawing-based processes to model-based processes, concluded that PMI has the potential to make many lifecycle processes run faster, with fewer errors, and at lower

cost, since STEP AP242 offers standards-based models that include the representation of PMI that is computer interpretable [5]. This is a major breakthrough that supports manufacturing's need for model-based computer-aided manufacturing (CAM) and coordinatemeasurement system (CMS) processes. STEP AP242 increases the effectiveness of modelbased enterprise (MBE) by enabling a common path for model-based definition (MBD) and model-based manufacturing (MBM) integration [6, 8].

Typical uses of STEP AP242 in industry are:

- computer-aided design (CAD) to CAD

- CAD to CAM and computer-aided inspection (CAI)

- LOTAR of design data

The industrial stakeholders currently driving changes to the standard are:

- Industrial original equipment manufacturers (OEMs) (e.g., aerospace, automotive)

- Defense manufacturing suppliers

- Government

Considerations regarding STEP implementation include:

- All commercially available geometry implementations use the EXPRESS language, ISO 10303-11 [9] and the EXPRESS-based file format, ISO 10303-21 [10]. There is a risk of limited future support as EXPRESS expertise diminishes. 
- STEP is widely adopted in all major CAD systems, but users must ensure that their computer-aided technologies (CAx) systems support the relatively new STEP AP242.

\subsection{ISO/ASTM 52915 (AMF)}

International Standards Organization (ISO)/American Society for Testing and Materials (ASTM) 52915:2016 [11] is an international standard that describes an interchange format for additive-manufacturing technology. The standard provides the specification for the Additive Manufacturing File Format (AMF), which is intended to replace the long-standing Stereolithography (STL) file format. STL only supports a surface mesh to define a part. In contrast, AMF supports the part's boundary representation and also includes support for color, texture, material specification, and substructures.

The ISO/ASTM 52915:2016 standard [11] claims AMF is backward compatible with STL $^{1}$ while also being extensible for future capabilities. The standard requires that all AMF files must conform strictly to the published Extensible Markup Language (XML) schema. Both, ISO and ASTM make a standard XML Schema Definitions (XSD) available for the AMF specification. The standard defines a minimum implementation for both the AMF file producer and consumer. The AMF file producer must, at a minimum, generate a compressed file with a single object and no additional properties. The AMF file consumer must, at a minimum, read a compressed file with a single object and ignore all additional properties.

Typical uses of AMF in industry are:

- CAD to CAD

- CAD to CAM

The industrial stakeholders currently driving changes to the standard are:

- Academia

- Government

- Industrial end-users

- Machine-tool manufacturers and solution providers

Considerations regarding AMF implementation include:

- The standard provides an implementation guide that includes a description of each AMF element, mathematical operation, and function.

- There is limited commercial vendor implementations of the standard. Support for the standard in CAD and CAM tools may be available in only the latest versions of the tools.

${ }^{1}$ The ISO/ASTM 52915:2016 standard allows a STL file to be converted directly to an AMF file. 


\subsection{ISO 6983 (G-code)}

ISO 6983-1:2009 [12] is an international standard that defines the data format to program position, line motion, and contouring control systems in the numerical control (NC) of machines. This data format is commonly known as G-code. G-code was created at MIT in the late 1950s and, like CAD, rose in popularity through the 1970s [13]. Today, G-code is the near-universal format for programming computer-based NC machines.

G-code is generated typically from a manufacturing plan using a CAM system. G-code files are defined using a standardized ASCII-based set of commands. Each line of the Gcode is a new command to the machine. Header information is standardized to support some traceability.

Typical uses of G-code in industry are:

- $\mathrm{CNC}$ control

- CNC operations

The industrial stakeholders currently driving changes to the standard are:

- $\mathrm{CNC}$ control manufacturers and solution providers

Considerations regarding G-code implementation include:

- Code logic and control is based on decades-old technology, when paper-tape / punch cards were used to numerically control machines. G-code limits the ability to handle highly complex geometries or to correct issues in-process.

- G-code is the only standard-based format for CNC programming, but machine-tool vendors have their own "flavors" of the standard and unique machine tool capabilities lead to data format variations between machines.

\subsection{ANSI MTConnect}

MTConnect [14] is an open-source, read-only data-exchange standard for manufacturing equipment and applications developed by the MTConnect Institute. It provides a standard domain model and a basic minimum infrastructure to enable the creation of structured, contextualized data for client applications. In this way, MTConnect enables semantic interoperability between different manufacturing systems and client applications. While other communication protocols may exist for data transfer, the information models defined in MTConnect provide a common vocabulary and structure created for manufacturingequipment data. Perhaps the most important type of data addressed by the standard is real and near-realtime data from the equipment (e.g., speed, position, program blocks). This data enriches the digital thread by providing information about the as-built condition of a part. 
The MTConnect standard defines four types of information models for manufacturing equipment: Devices, Streams, Assets, and Interfaces [14]. Devices provides a representation of the physical and logical configuration for a piece of manufacturing equipment and the definition of data that may be reported by that equipment. Streams provides a specification for time-stamped data values returned by a piece of manufacturing equipment. Assets provides models of items that are not considered integral to a piece of manufacturing equipment but are used in the manufacturing process, e.g., cutting tools. Interfaces models the information needed to coordinate actions between different pieces of manufacturing equipment.

Users access the data and information provided by the information models in MTConnect through the Agent [14]. The Agent implements the MTConnect protocol and generates the relevant response document, which is traditionally in XML. MTConnect only standardizes communication between the Agent and an application. The Agent returns data only when requested by an application. The Agent acts as an Hypertext Transfer Protocol (HTTP) server and uses Representational State Transfer (REST) when interacting with any data source. In other words, the data source is responsible for sending state updates to the Agent.

Typical uses of MTConnect in industry are:

- Overall equipment effectiveness and utilization

- Process monitoring

- Condition monitoring

The industrial stakeholders currently driving changes to the standard are:

- Manufacturing equipment vendors

- Manufacturing solution providers and developers

- Industrial end-users (e.g., aerospace, defense)

Considerations regarding MTConnect implementation include:

- Deploying one agent for the entire shop floor versus deploying one agent per machine on the shop floor (e.g., one agent per machine has higher maintenance overhead but does not requiring turning off data collection for all machines during maintenance, using one Agent for multiple machines may present challenges with the Agent's buffer)

- Different communication protocols may be used for transporting the MTConnectcompliant information (e.g., Message Queuing Telemetry Transport (MQTT), Open Platform Communications United Architecture (OPC-UA))

- Curation of data may occur at various times of collection and transport (e.g., curate at machine edge, curate aggregation of data for a shop, curate date by part) 


\subsection{ANSI/DMSC Quality Information Framework}

The Quality Information Framework (QIF) [15] is an ANSI-accredited standard sponsored by the Dimensional Metrology Standards Consortium (DMSC). QIF defines an integrated set of XML information models that enable the effective exchange of metrology data throughout the entire metrology process. QIF handles feature-based dimensional metrology, quality measurement planning, first article inspection, and discrete quality measurement. QIF supports defining or importing the product definition and reusing data for inspection planning, execution, analysis, and reporting.

QIF uses terminology and semantics from the inspection world to represent the various elements in the QIF specification. QIF uses XSD to normalize the information models. The standard organizes QIF XSDs into six application areas for metrology: (1) MBD, (2) Rules, (3) Resources, (4) Plans, (5) Results, (6) Statistics. The standard combines the MBD (containing the product definition) with measurement rules and resources definitions to generate a plan. The plan is then executed and the results are captured. Multiple results are combined to generate statistics. QIF does not perform the task of statistics and the other metrology methods. Instead, QIF enables the ability to put raw inspection data into a quality context that is computer-processable.

Typical uses of QIF in industry are:

- Capturing and transmitting inspection results between organizations

- Describing available measurement assets

The industrial stakeholders currently driving changes to the standard are:

- Industrial end-users (e.g., aerospace, defense)

- Metrology solution providers

- Government

Some general implementation considerations are:

- All parts of QIF are not implemented by all commercial solution providers. QIF results is the most adopted part of the standard.

- Persistent ID of features and characteristics are optional in the QIF standard, but required for full traceability of quality across the product lifecycle.

\subsection{ISO 32000 and ISO 14739 (PDF/PRC)}

The Portable Document Format (PDF) [16] and Product Representation Compact (PRC) [17] International Standards are often combined into technologies for visualizing productdefinition data. PDF/PRC is commonly referred to as a 3D PDF. While there are several flavors of 3D PDF, the combination of PRC embedded in a PDF document is emerging as 
the industry recommended practice. PDF/PRC enables the display of 3D product definition in any PDF reading software that conforms to the standard. Using PDF/PRC enables effective and efficient visualization of product data throughout the lifecycle for humanconsumption. A significant amount of metadata may be included in the PRC and additional metadata can be included in the PDF document via XML namespaces wrapped in a Extensible Metadata Platform (XMP) package.

Typical uses of PDF/PRC in industry are:

- Visualization of design data without the use of CAD tools

- Container for transmitting a technical data package (TDP)

The industrial stakeholders currently driving changes to the standard are:

- PDF solution providers

- Industrial end-users (e.g., aerospace, defense)

- Government

Considerations regarding PDF/PRC implementation include:

- PRC includes a boundary representation (BREP), but no commercially available manufacturing or quality systems interact directly with the BREP in manufacturing or quality processes.

\section{Example: NIST SMS Test Bed}

The NIST SMS Test Bed provides an example of a network solution that was developed using the guidance in this document to support the collection, curation, and re-use of manufacturing data from the NIST Fabrication Technology Office (FTO). A description of this solution and how it was developed can be found by consulting Ref. [18]:

Citation: Helu M, Hedberg Jr T (2020) Connecting, deploying, and using the smart manufacturing systems test bed. National Institute of Standards and Technology, Report NIST AMS 200-2. doi: 10.6028/NIST.AMS.200-2

\section{Acknowledgments}

The authors wish to thank Allison Barnard Feeney and Vijay Srinivasan from NIST, Paul Huang from the Office of Naval Research Laboratory, Will Sobel from VIMANA, and Russell Waddell from the Association for Manufacturing Technology for their comments and input to this report. The authors prepared this report as partial fulfillment to the requirements covered by the reimbursable agreement N0001418IP00053 between NIST and the 
Office of Naval Research Laboratory.

\section{References}

[1] Bernstein WZ, Hedberg Jr TD, Helu M, Feeney AB (2017) Contextualising manufacturing data for lifecycle decision-making. International Journal of Product Lifecycle Management 10(4):326-347. https://doi.org/10.1504/IJPLM.2017.090328

[2] Helu M, Weiss B (2016) The current state of sensing, health management, and control for small-to-medium-sized manufacturers. Proceedings of the ASME 2016 Manufacturing Science and Engineering Conference, , . https://doi.org/10.1115/ MSEC2016-8783

[3] Helu M, Hedberg Jr T (2015) Enabling smart manufacturing research and development using a product lifecycle test bed. Procedia Manufacturing 1:86-97. https://doi.org/10.1016/j.promfg.2015.09.066

[4] International Standards Organization (2014) Industrial automation systems and integration - product data representation and exchange - part 242: application protocol: managed model-based 3D engineering.

[5] Barnard Feeney A, Frechette SP, Srinivasan V (2015) A portrait of an ISO STEP tolerancing standard as an enabler of smart manufacturing systems. Journal of Computing and Information Science in Engineering 15(2):21001. https://doi.org/10.1115/ 1.4029050

[6] Trainer A, Hedberg Jr T, Barnard Feeney A, Fischer K, Rosche P (2016) Gaps analysis of integrating product design, manufacturing, and quality data in the supply chain using model-based defintion. ASME 2016 11th International Manufacturing Science and Engineering Conference - Volume 2: Materials; Biomanufacturing; Properties, Applications and Systems; Sustainable Manufacturing (ASME), Vol. 2, p V002T05A003. https://doi.org/10.1115/MSEC2016-8792

[7] Hedberg Jr T, Lubell J, Fischer L, Maggiano L, Barnard Feeney A (2016) Testing the digital thread in support of model-based manufacturing and inspection. Journal of Computing and Information Science in Engineering 16(2):021001. https://doi.org/ 10.1115/1.4032697

[8] Fischer A, Arthurs G (2015) INCOSE 2015 MBSE workshop breakout session. Available at http://www.omgwiki.org/MBSE/doku.php?id=mbse:incose $\left\{_{-}\right\} \operatorname{mbse}\left\{{ }_{-}\right\} \operatorname{iw}\left\{{ }_{-}\right\}$ 2015: breakout $\{$ _ $\}$ out $\left\{{ }_{-}\right\}$session $\left\{_{-}\right\}$model $\left\{\left\{_{-}\right\}\right.$lifecylce $\{-\}$mgmt.

[9] International Standards Organization (2004) Industrial automation systems and integration - product data representation and exchange - part 11: Implementation methods: The express language reference manual.

[10] International Standards Organization (2016) Industrial automation systems and integration - product data representation and exchange - part 21: implementation methods: clear text encoding of the exchange structure.

[11] International Standards Organization, American Society for Testing and Materials 
(2016) Standard specification for additive manufacturing file format (AMF) version 1.2.

[12] International Standards Organization (2009) Automation systems and integration numerical control of machines - program format and definitions of address words part 1: data format for positioning, line motion and contouring control systems.

[13] Suh SH (2008) Theory and design of cnc systems (Springer, London), .

[14] MTConnect Institute (2018) MTConnect standard version 1.4.0 (ANSI/MTC1.42018). Available at https://www.mtconnect.org/s/ANSI _ $\}$ MTC1 $\left\{_{-}\right\} 4-2018 . p d f$.

[15] Digital Metrology Standards Consortium (2018) QIF standard version 3.0 (ANSI/DMSC QIF 3.0). Available at https://qifstandards.org/qif-download.

[16] International Standards Organization (2008) Document management - portable document format - part 1: PDF 1.7.

[17] International Standards Organization (2014) Document management - 3D use of product representation compact (PRC) format - part 1: PRC 10001.

[18] Helu M, Hedberg Jr T (2020) Connecting, deploying, and using the smart manufacturing systems test bed (National Institute of Standards and Technology), Report NIST AMS 200-2. https://doi.org/10.6028/NIST.AMS.200-2 